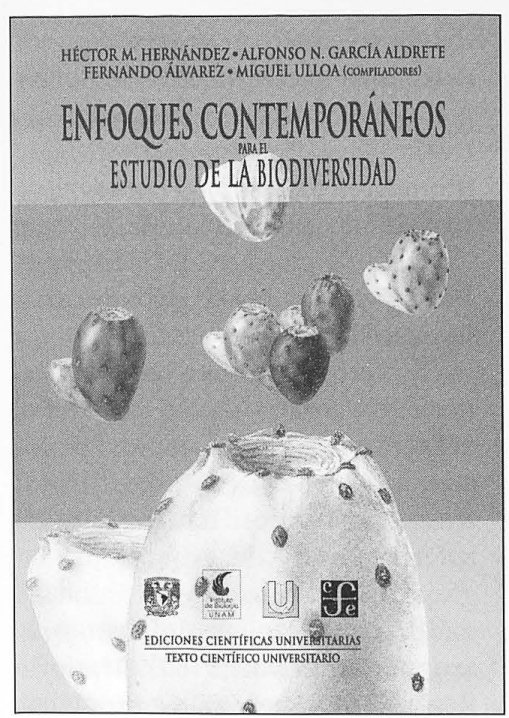

\section{A LA RECHERCHE DU TEMPS PERDU}

\author{
Hernández, H.M., A.N. García Aldrete, \\ F. Álvarez y M. Ulloa (COMPILAdores)
}

$\mathrm{E}$ n 1999, para celebrar los 70 años de la fundación del Instituto de Biología, se llevó a cabo un simposio sobre biodiversidad en el que "se expusieron y debatieron diversas líneas de investigación centrales para la comprensión de los organismos vivos" (P. Raven, en el prólogo del libro). El presente volumen es el resultado de estos trabajos. El estilo de las presentaciones es interesante, ya que en muchos casos son coautorías entre un investigador extranjero líder en su campo y un investigador del Instituto de Biología, aunque en la mayor parte de los casos el extranjero es el primer autor. Realmente, el libro se puede considerar como un extraordinario y valiente ejercicio de reflexión por parte del Instituto de Biología para enfocar, o en su caso reorientar, sus líneas de investigación a la luz de un mundo cambiante, donde han surgido nuevas herramientas y metodologías para el estudio de la diversidad biológica, pero al mismo tiempo la biodiversidad está desapareciendo a niveles previamente inimaginables.
Como el título de la obra lo indica, consiste en diferentes revisiones sobre el estudio de la diversidad biológica, abarcando gran cantidad de ideas, estilos, organismos y preocupaciones. Ordenando de manera más o menos jerárquica, el primer nivel del estudio de la biodiversidad podría ser el estudio de la variación intraespecífica. Dentro de esta importante área de estudio, sólo tenemos un artículo, el de David Spooner y Sabina Lara-Cabrera, enfocado a revisar estudios recientes utilizando marcadores moleculares en plantas cultivadas. Esta es un área con una larga y exitosa tradición, como lo demuestran la gran cantidad de referencias que reseñan, a pesar de haberse concentrado solo en los estudios publicados desde 1994 . Desafortunadamente, me da la impresión que los autores se ahogaron en el mar de información que recopilaron, y de las ocho páginas de tablas con los estudios que encontraron, lo que logran rescatar son una serie de 15 "preguntas" ejemplares que se han atacado en algunos de estos trabajos. Real- mente, hubiera sido más útil que describieran patrones, como lo ha hecho para plantas silvestre en un larga serie de artículos James Hamrick (uno de los más recientes es Hamrick y Godt, 1997), o los de Doebley (1989) para plantas cultivadas, o si hubieran tomado un grupo más definido de plantas, como las solanáceas americanas que ha estudiado el mismo Spooner.

Como segundo posible nivel jerárquico en el estudio de la biodiversidad podríamos considerar el estudio de la diversidad de características y adaptaciones en grupos de organismos. Dos trabajo se podrían ubicar en ésta categoría. Por una lado, tenemos la revisión sobre la diversidad anatómica en las cactáceas, realizada James Mauseth. Estas diferentes y extraordinarias características son interpetadas por Mauseth como adaptaciones, aunque realmente serían interesante que incluyera observaciones experimentales o análisis estilo método comparativo (ver adelante). Así, Mauseth lo podríamos ubicar en la escuela "clásica" de botánicos evo- 
lutivos, los cuales se dedicaron a describir grandes patrones que eran interpretados como evidencias de adaptación y selección natural. Es interesante notar que del total de las referencias (24), él es autor o coautor de todas. Me cuesta trabajo pensar que nadie más haya hecho contribuciones relevantes a la anatomía, fisiología, ecología o evolución de las Cactaceae (ver por ejemplo Gibson y Nobel, 1986).

En este segundo nivel también podemos colocar al capítulo de Kent Holsinger, quien analiza las características relacionadas con la biología reproductiva en plantas. En términos generales, desde $\mathrm{Da}$ rwin los botánicos se han intrigado por la gran diversidad de sexualidades que presentan las plantas, y han tratado de interpretarlas adaptativamente. Sin embargo, buena parte del tiempo los botánicos se concretaron, como Mauseth para la anatomía de las Cactaceae, en describir grandes patrones, como por ejemplo de los síndromes de polinización (Faegri and Van der Pijl, 1979), y no es hasta que David Lloyd en 1965 propone buscar con modelos y experimentos evidencias de ventajas a nivel individuo de las características sexuales de las plantas. Holsinger presenta un interesante estudio, al sugerir que parte los problemas en el avance de este campo se deben a que los estudios se han realizado por un lado por ecólogos y por otro por genetistas de poblaciones, con diferentes énfasis y terminologías, y poco o nulo contacto entre ambos y que no se han puesto de acuerdo para resolver los problemas que requiere de ambas experiencias. Los problemas en particular que trata su revisión son en relación con las condiciones en las cuales se favorece la autofertilización y el papel de la depresión por edogamia (ver Eguiarte et al. 1999 y Eguiarte y Piñero 1999). Entre los aspectos más atractivos del artículo se encuentran la revisión de estudios recientes utilizando secuencias de ADN, que han ayudado a entender mejor la importancia relativa de fecundación cruzada, la selección a favor autofertilización y la depresión por endogamia, y algunos modelos recientes, como el del "mutational meltdown" de Lynch y colaboradores (Gabriel et al., 1993): sin sexualidad, en poblaciones finitas por necesidad se acumulan las mutaciones deletéreas (esto es el "Muller ratchet", ver Eguiarte $e$ t al., 1999), lo que a su vez hace que se reduzca el tamaño efectivo de las poblaciones y se fijen más rápido más mutaciones deletéreas. Este ciclo se repite de manera automática, así que se acumulan más y más mutaciones deletéreas a medida que se reduce progresivamente el tamaño poblacional, hasta que finalmente se extingue el linaje endogámico/asexual. La revisión de Hoslinger es excelente, aunque similar a muchas otras en la literatura, incluyendo una muy reciente por el mismo Holsinger, que revise en estas páginas en el número anterior (Holsinger, 2000; Eguiarte, 2001; ver también por ejemplo Eguiarte et al., 1999).

Un tercer nivel jerárquico dentro del estudio de la diversidad sería describir el número de especies. En esta categoría podemos ubicar varios de los capítulos. Así por ejemplo, Sandra Knapp, Gerrit Davidse y M. Sousa nos platican de sus experiencias y expectativas en relación al monumental estudio de la Flora Mesoamericana. El entusiasmo de los autores por su proyecto, y la calidad y magnitud del mismo es innegable. Buena parte del artículo se lo dedican o hacen referencia al supuesto sitio en el internet del proyecto de la Flora Mesoamericana (www. fm), pero desafortunadamente esta dirección corresponde la página turística de la Federación de Estados de la Micronesia (bueno, cuando menos no es un sitio pornográfico...). Aparentemente la dirección correcta era, el día que logré encontrarla, www. mobot.org/ mobot/ $\mathrm{fm}$, pero no puedo garantizar que siga siendo (tampoco encontré un "link" en la página del Instituto de Biología o en la del Missouri Botanical Garden).

Si bien la manera obvia de tener el inventario de las especies es colectando, desde hace algunos años varios biólogos relacionados a la conservación han tratado de inferir el total de especies de un grupo taxonómico o de un ambiente dado (ver por ejemplo el notorio artículo de Erwin, 1982). Marjorie Reaka-Kudla se dedica a esta labor en relación a la diversidad que habita a los arrecifes de coral. Aunque se han descrito unas 90 mil especies en ellos, Marjorie calcula que en total existen entre 1 y 5 millones de especies coralíferas. Al mismo tiempo, las tasas de su destrucción son impresionantes, debidas a gran cantidad de factores. En contraste, Jean Lodge en vez de intentar una estimación del total de hongos en el planeta, lo que hace es una crítica de estos estimados, especialmente los basados en proyecciones de lo que se conoce en ambientes templados (usualmente léase Inglaterra) al resto del mundo. Estas estimaciones en hongos parecen ser una industria activa, ya que si no mal recuerdo una ya agració las páginas de esta revista (Guzmán, 1994). Este número se ha calculado para los hongos generalmente entre 1 y 1.5 millones de especies en el planeta, aunque otros estudios sugieren que podría ser mucho más, cuando menos de 13.5 millones de especies, si se siguen los patrones reportados para los hongos que son parásitos de insectos. Ella fundamenta sus críticas a la luz de su experiencia con hongos tropicales, especialmente los ascomicetos xiliriaceos endófitos tropicales, 
que aparentemente tienen distribuciones muy amplias y baja especificada.

Otro problema relacionado con la diversidad a nivel inventario de especies es el de conocer el área de distribución de cada una de estas especies. Dado que no vamos a poder tener datos de colecta de las especies en todos los lugares donde se ha colectado, se han propuesto varios métodos. Víctor Sánchez Cordero, Townsend Peterson y Patricia Escalante revisan estos métodos, con énfasis en los llamados "algoritmos genéticos", en particular el llamado GARP, y dan ejemplos de uso en problemas de conservación, con datos de aves y mamíferos mexicanos. En particular destaca la mención del estudio de Peterson y Navarro-Sigüenza (1999) donde al aplicar sus conceptos "filogenéticos" de especies (elevar las subespecies y lo linajes que ellos creen independientes al rango de especies) cambian los lugares con mayor incidencia de endemismo en aves con respecto a las zonas que resultan según las definiciones "normales" de especie. El problema radica en el excesivo peso que se la ha dado a las especies en la biología de la conservación, y sugiero que se hagan estudios detallados de todas estas supuestas "especies" para ver si realmente constituyen poblaciones geneticamente diferenciadas. Por último hacen un llamado a que se haga accesible el acceso electrónico ilimitado a las colecciones científicas, llamado que esperemos haga suyo el Instituto de Biología a la mayor brevedad.

Tres trabajos tratan sobre un cuarto nivel de la biodiversidad, la diversidad de grupo de organismos. David Green, Robert Carrol y Víctor-Hugo Reynoso presentan un ambicioso trabajo en el que tratan de entender la crisis actual de la declinación de muchas de las poblaciones de anfibios partir del análisis de su historia evolutiva, en particular al incluir sus patrones de extinción en el pasado. Sin embargo, los grupos de anfibios que se extinguieron en la antigüedad eran filogenética, ecológica, y morfológicamente muy distantes de los que viven hoy día, y las causas de su extinción seguramente también fueron diferentes a las actuales. Por lo anterior no queda nada claro que las lecciones del pasado sean útiles, ni que nadie tenga una idea general o muy clara de porqué están declinado las poblaciones de estos organismos en la actualidad. El capítulo presenta el error de traducción más grave del libro, al llamar al "Ne" como el "tamaño real de las poblaciones". $\mathrm{N}_{\mathrm{e}}$ es el "tamaño efectivo", un parámetro central en la genética de poblaciones, y claramente definido por Sewall Wright. El $\mathrm{N}_{\mathrm{e}}$ lo que hace es convertir el tamaño censal a un número que nos dice cómo se va a perder la variación genética e incrementar la varianza en las frecuencias alélicas entre poblaciones debido a la deriva génica (ver Eguiarte, 1990, Eguiarte et al., 1993) y que siempre es menor que al tamaño censal (o sea, el "real", ver Eguiarte et al. 1994).

Sobre grupos particulares de organismos también tenemos al capítulo sobre líquenes de María de los Angeles Herrera-Campos y Thomas Nash. Los líquenes son superorganismos formados por un hongo que se "aprovecha" de una alga verde o una cianobacteria. Esto genera problemas para la taxonomía y para entender su biología evolutiva. Los taxónomos se han enfocado en estudiar al hongo y en base a él clasifican a los líquenes, con el problema que en algunos casos un mismo hongo con diferente simbiontes generan líquenes con morfología muy diferentes. Así, existen muchas dudas y controversia en el grupo sobre las definiciones de especies, los posi- bles caracteres para su estudio, su filogenia, y el grado de flujo génico y sexualidad, y sobre los marcadores moleculares adecuados para resolver estos problemas. Yo sugeriría que retomara la amplia y exitosa experiencia del uso de la genética de poblaciones y evolución molecular en bacterias, con métodos multilocus para definir el grado de clonalidad/sexualidad y aislamiento genético de los linajes (ver por ejemplo, Souza et al., 1993, 1994 y 1999) y que analizaran con marcadores moleculares para encontrar si las "víctimas" (algas o bacterias) covarían filogenéticamente con los hongos o no, como se sugiere en el esquema $4 \mathrm{del}$ capítulo de Pellmyr et al. (en éste volúmen), o según la teoría propuesta por Souza y Eguiarte (1998) para bacterias, sólo cambiando cromosoma por hongo y plásmido por alga/cianobacteria.

Un ambicioso análisis sobre la biología de los parásitos es presentado por Daniel Brooks, Virgina León-Regagnon y Gerado PérezPonce de León. El tema es fascinante, y se conecta al siguiente grupos de capítulos, pero desafortunadamente el énfasis del manuscrito es en demostrar la importancia del estudio de los parásitos para la diversidad en base a documentos como el programa Diversitas, o las Agenda Sistemática del Año 2000. Creo que el énfasis es más bien político que académico, lo cual es una lástima, ya que varios de los ejemplos filogenéticos tratados someramente son fascinantes.

Una de las formas más populares y poderosas de visualizar la diversidad es usando filogenias. El resto de los capítulos el libro tratan de la diversidad a este nivel. Kevin Nixon y Helga Ochoterena revisan su versión de las ideas y métodos de la reconstrucción filogenética por el método de parsimonia. Indudablemente los autores le han dedicado mucho tiempo a pensar 
sobre el problema y a utilizar las metodologías en diferentes análisis. Sin embargo, dogmatizan de manera confusa contra otros métodos de reconstrucción filogenética (como los modelos de maximum likelihood, erróneamente traducido como "máxima probabilidad", o el de neighbor-joining), o insisten que se deben de usar siempre todos los datos disponibles en el análisis filogenético (evidencia total), juntando tanto caracteres morfológicos y moleculares con las adaptaciones que quiera estudiar el biólogo evolutivo, idea no muy apreciada por otros biólogos, por su sabor a circularidad y por el problema de las diferencias en la tasa de evolución y convergencia por selección natural de las adaptaciones. Todas estas cuestiones distan mucho de estar zanjadas y en el volumen otros autores usan enfoques contrarios. En particular, en su excelente aunque breve capítulo Olle Pellmyr et al. revisan los conceptos de coevolución, a la luz del mosaico coevolutivo de Thompson, y para eso utilizan datos de filogenias moleculares de polillas de la Yucca (Prodoxus, Tegeticula), utilizando métodos con el neighbor-joining y mapeando caracteres evolutivo y morfológicos en dichas filogenias. Debo señalar que los tiempos de origen que sugieren (figura 3) de varios procesos que discuten, como el de la colonización de las yucas por las polillas, son cuando menos del doble de antigüedad que los que yo he calculado con datos de las secuencias de Agavaceae (ver Eguiarte, 1995; Eguiarte et al. 2000).

Otros dos artículos usan las filogenias. Gustavo Hormiga y Jonathan Coddington, usando una filogenia previa de las arañas que tejen redes orbiculares, reconstruyen los ancestros del tamaño de machos y hembras, utilizando los métodos implementados en el programa MacClade de los Maddison
(1993). En estas arañas es común un dimorfismo de tamaño, y se ha considerado tradicionalmente que las machos han evolucionado a ser "enanos". Sin embargo, al hacer el análisis encuentran que en varios casos lo que sucede es que las hembras son las que aumentan de tamaños. Desafortunadamente, no propone ninguna hipótesis de por qué sucede esto tantas veces en la familia, ni exploran en qué condiciones ecológicas sucede.

Por otra parte, Wayne Maddison y Tila Pérez contribuyen con un original ensayo, donde revisan varias ideas y sobre filogenias y biodiversidad. Una buena parte del artículo esta dedicado a cómo analizar adaptaciones (que llaman "apomorfías heróicas") en el contexto filogenético, junto con algunos análisis sofisticados en el que el primer autor ha trabajado y que se ubican dentro de lo que se ha llamado el análisis de "método comparativo" (ver Morales, 2000). Sin embargo, muchas de las metáforas que usan en el capítulo, como la "curva espacio tiempo", considerando la perspectiva ecológica como el "plano newtoniano" de la diversidad, y el de la filogenia como el "plano einsteniano", me parece que confunden más que ayudar, lo mismo que algunos de los tortuosos ejemplos sobre arañas que presenta, como el de los quiasmas cromosomales y la determinación sexual en arañas saltadoras.

Paradójicamente, la conclusión del libro la proporciona una contribución singular de Paul Ehrlich. Buena parte de su capítulo lo dedica a la reconstrucción filogenética, pero lo que hace es más bien criticar a los cladistas y su dogmatismo, y a señalar que él considera un ejercicio inútil, estilo labor de un monje medieval, los esfuerzos por reconstruir la filogenia de grupos obscuros (y nos podría venir a la mente los parásitos de Brooks et al.). Él sugiere trabajar bien los grupos "importantes", como aves, mamíferos y mariposas, y a partir de sus datos, tratar de encontrar patrones relevantes, especialmente para la conservación. El capítulo está estructurado como una crítica a las tres misiones que señala la Systematics Agenda 2000, la misma que ocupaba a Brook $e t$ al., que resumiendo son: 1] Inventariar la diversidad, 2] Obtener la filogenia del total del árbol de la vida (o sea, de todos los organismos), 3] Organizar la información de estos estudios. Sus ideas son interesantes y posiblemente correctas en muchos puntos, pero el estilo es muy controversial y resulta anticlimático considerando el resto del libro.

Como conclusión, quiero decir que esta es una obra interesante y actualizada, que toca varios de los tópicos y controversias contemporáneas sobre el estudio de la diversidad biológica, y estoy seguro que si su precio es accesible y su distribución adecuada, será un "milestone" en la biología de México y América Latina, y una obra importante para la docencia a nivel licienciatura y posgrado. Adicionalmente, puede cumplir con uno de los objetivos del simposio, o sea de alguna forma servir para definir el nicho y renovar la misión del Instituto de Biología en un mundo científica y ecológicamente cambiante. A mi me hubiera gustado ver una presencia más activa de los editores, organizando los trabajos con un orden lógico (parece que se dejaron en el orden como presentaron), e introduciendo los artículos y las secciones con revisiones actualizadas. Por otro lado, los autores no parecen conocer o apreciar el trabajo de los demás, ya que no recuerdo ni una referencia cruzada a los capítulos de lo otros autores. Además, me hubiera gustado ver las perspectivas de los editores sobre estos problemas, sus comentarios y sus ideas, y creo 
que se hubiera podido invitar a algunos líderes nacionales no asociados al Instituto de Biología. Por otra parte, y se podría interpretar de muchas manera, el libro sólo incluye un índice taxónomico, y creo que hubiera valido la pena más bien tener uno temático.

\section{Referencias}

Doebly, J. 1989. Isozymic evidence and the evolution of crop plants. en D.E. Solits y P.S. Soltis, eds. Isozymes in plant biology. Dioscoride press, Portland, Oregon, EUA.

Eguiarte, L.E. 1990. Genética de poblaciones de Astrocaryum mexicanum Liebm. en Los Tuxtlas, Veracruz. Tesis de doctorado. Centro de Ecología/UACPyP del CCH, C.U., Universidad Nacional Autónoma de México, México, D.F, México.

Eguiarte, L.E. 1995. Hutchinson (Agavales) vs. Huber y Dahlgren (Asparagales): análisis moleculares sobre la filogenia y evolución de las familias Agavaceae sensu Hutchinson dentro de las monocotiledóneas. Boletín de la Sociedad Botánica de México. 56:45-56.

Eguiarte, L.E. 2001. Cincuenta años de botánica evolutiva, Reseña del libro: Ayala F.J., W. M. Fitch y M.T. Clegg, editores. 2000. Variation and evolution in plants and microorganisms: Towards a new synthesis 50 years after Stebbins. National Academy of Science, Washington, D.C., E.U.A.

Eguiarte, L. E., A. Búrquez, J. Rodríguez, M. Martínez-Ramos, J. Sarukhán y D. Piñero. 1993. Direct and indirect estimates of neighborhood and effective population size in a tropical palm, Astrocaryum mexicanum. Evolution 47:75-87.

Eguiarte, L. E., V. Parra y F. Vargas. 1994. Biología reproductiva y tamaño efectivo en Echeveria gibbiflora: Un homenaje a Sewall Wright, en A. Rojo (ed.) Reserva Ecológica del de biología evolutiva, Facultad de Ciencias, UNAM. Pedregal de San
Angel: Ecología, Historia Natural y Manejo. UNAM. págs 187-204.

Eguiarte, L. E., J. Nuñez-Farfán, C. Domínguez y C. Cordero. 1999. Biología evolutiva de la reproducción en plantas. en Nuñez-Farfán J. y L. E. Eguiarte (editores) La Evolución Biológica. Facultad de Ciencias, Instituto de Ecología, UNAM, CONABIO, págs. 117-152.

Eguiarte, L. E. y D. Piñero. 1999. Génetica de la conservación: leones vemos, genes no sabemos, en NuñezFarfán J. y L. E. Eguiarte (editores) La Evolución Biológica. Facultad de Ciencias, Instituto de Ecología, UNAM, CONABIO, págs. 371-398.

Eguiarte, L.E., A. Silva y V. Souza. 2000. Biología evolutiva de la familia Agavaceae: biología reproductiva, genética de poblaciones y filogenia. Boletín de la Sociedad Botánica de México. 166:131-150.

Erwin, T.L. 1982. Tropical forest: their richness in Coleoptera and other arthropod species. Coleopterist Bulletin 36:74-76.

Faegri K. y L. van der Pijl. 1979. The Principles of pollination ecology. Pergamon Press. Oxford, England.

Gabriel, W., M. Lynch y R. Bürger. 1993. Muller's ratchet and mutational meltdown. Evolution 47:1744-1757.

Gibson y Nobel, 1986. The cactus primer. Harvard University Press, Cambridge, Massachusetts EUA.

Guzmán, G. 1994. Las colecciones de hongos en México y su problemática en la biodiversidad del país. Boletin de la Sociedad Botánica de México 55:35-37.

Hamrick J.L. y M.J.W. Godt. 1997. Effects of life history traits on genetic diversity in plant species. en Silvertown, J. M. Franco y J.L. Harper, eds. Plant life hisotries: ecology, phylogeny and evolution. Cambridge University Press.

Holsinger, K.E. 2000. Reproductive systems and evolution of vascular plants. En Ayala F.J., W. M. Fitch y M.T. Clegg, eds. 2000. Variation and evolution in plants and microorganisms: Towards a new synthesis 50 years after Stebbins. National Academy of Science, Washington, D.C., E.U.A.

Lloyd, D.G. 1965. Evolution of selfcompatibility and racial differentation in Leavenwothia (Crucifera). Contributions of the Gray Herbarium 195:3-133.

Maddison W.P. y D.R. Maddison. MacClade. Analysis of phylogeny and character evolution. Version 3.04. Sinauer Associates, Suderland, Massachusetts, EUA

Morales, 2000. El método comparativo en ecología vegetañ. Boletin de la Sociedad Botánica de México 66:37-51.

Peterson, A.T., y A.G. Navarro-Sigüenza 1999. Alternate species concepts as bases for determining priority conservation areas. Conservation Biology 13:427-431.

Souza, V., T. T. Nguyen, D. Hudson, D. Piñero and R. E. Lenski (1992). Hierarchical analysis of linkage disequilibrium in Rhizobium populations: Evidence for sex?. Proceedings of the National Academy of Science USA, 89, 8389-8393

Souza, V., L. Eguiarte, G. Avila, R. Cappello, C. Gallardo, , J. Montoya y D. Piñero 1994. Genetic structure of Rhizobium etli biovar phaseoli associated with wild and cultivated bean plants (Phaseolus vulgaris and $P$. coccineus) in Morelos, Mexico. Applied and Environmental Micorbiology 60:1260-1268.

Souza, V. y L. E. Eguiarte. 1997. Bacteria gone native vs. bacteria gone awry?: Plasmidic transfer and bacterial evoution. Proc. Natl. Acad. Sci. USA. 94:5501-5503.

Souza V., M. Rocha. A. Valera y L. E. Eguiarte. 1999. Genetic structure of natural populations of Escherichia coli in wild hosts on different continents. Applied and Environmental Microbiology 65: 3373-3385.

\section{Luis E. Eguiarte}

Departamento de Ecología Evolutiva, Universidad Nacional Autónoma de México, Apartado Postal 70-275, CP 04510, C.U. Coyoacán, México D.F., México.fruns@servidor.unam.mx 